\title{
Introduction to Quasielastic Neutron Scattering
}

\section{Journal Article}

\section{Author(s):}

Embs, Jan Peter; Juranyi, Fanni; Hempelmann, Rolf

Publication date:

2010

Permanent link:

https://doi.org/10.3929/ethz-b-000023188

\section{Rights / license:}

In Copyright - Non-Commercial Use Permitted

\section{Originally published in:}

Zeitschrift für physikalische Chemie 224(1-2), https://doi.org/10.1524/zpch.2010.6090 


\title{
Introduction to Quasielastic Neutron Scattering
}

\author{
By Jan Peter Embs ${ }^{1}$, Fanni Juranyi ${ }^{1}$, and Rolf Hempelmann ${ }^{2, *}$ \\ ${ }^{1}$ Physical Chemistry, Saarland University, Laboratory for Neutron Scattering, ETH Zurich, \\ Paul Scherrer Institut, 5232 Villigen-PSI, Switzerland \\ ${ }^{2}$ Physical Chemistry, Saarland University, PB 151150, 66041 Saarbrücken, Germany
}

(Received November 16, 2009; accepted November 27, 2009)

\section{QENS / Diffusion / Neutron Instrumentation}

\begin{abstract}
This tutorial introduction has been written for people who are not specialized in neutron scattering or in other scattering methods but who are interested and would like to get an impression and learn about the method of Quasielastic Neutron Scattering (QENS). The theoretical (scattering process) as well as the experimental basics (neutron sources, neutron scattering instruments, experimental periphery) are explained in a generally understandable way, with only the most essential formulas. QENS addresses the stochastic dynamics in condensed matter, and it is pointed out for which problems and for which systems in condensed matter research QENS is a powerful method. Thus sufficient information is provided to enable non-experts to think about their own QENS experiment and to understand related literature in this area of research.
\end{abstract}

\section{Introduction}

In the non-specialized scientific community the term neutron scattering is usually associated with Bragg peaks and structure determination and thus with neutron diffraction and neutron diffractograms, i.e. with plots of neutron scattering intensity versus the scattering angle $2 \theta$. Important and well-known is Bragg's scattering equation

$$
n \lambda=2 d \sin (\theta)
$$

where $\mathrm{n}$ is the diffraction order, $\lambda$ the wavelength of the radiation, $d$ the lattice plane spacing and $2 \theta$ the scattering angle. The scattering angle, i.e. the measured quantity, does not only depend on $d$, the property of the sample system, but also on the experimental parameter $\lambda$. Therefore, instead of recording the scattering intensity versus the scattering angle, it makes sense to record versus the momentum transfer $\hbar Q$ of elastically scattered neutrons, with

\footnotetext{
* Corresponding author. E-mail: r.hempelmann@mx.uni-saarland.de
} 


$$
Q=\frac{4 \pi}{\lambda} \sin (\theta)
$$

In comparison to this well-known technique, quasielastic neutron scattering (QENS), the topic of the present special volume of Zeitschrift für Physikalische Chemie, is clearly less commonly used. In a QENS experiment the intensities are measured as a function of both, scattering angle $2 \theta$ and energy transfer between neutrons and sample, $E=\hbar \omega=\hbar \omega_{i}-\hbar \omega_{f}=E_{i}-E_{f}$, where $E_{i}$ and $E_{f}$ denote initial and final neutron energy, respectively; this allows for an investigation of the dynamics of a system. QENS (see [1-4]) investigates small energy exchanges peaked at $E=0$. It can be used to study stochastic motions in condensed matter, e.g. rotational diffusion [2] and translational diffusion [3]. Diffusion is not quantized, and therefore the energy transfer can be infinitesimally small. However in the case of periodic motions like librations or vibrations, the energy is transferred in quanta, thus giving rise to inelastic scattering at certain energy transfers. In general, diffusion and vibration are considered as decoupled motions in good approximation, therefore vibrations effect only the intensity of the quasielastic scattering.

Neutrons are electrically neutral and therefore do not interact with the electrons of the atoms/molecules in condensed matter, but are scattered at their nuclei. Therefore QENS belongs to the nuclear methods in condensed matter physics [5] all of which are powerful only for few nuclei: NMR works very well for ${ }^{1} \mathrm{H},{ }^{2} \mathrm{H},{ }^{13} \mathrm{C},{ }^{29} \mathrm{Si}$, Mössbauer spectroscopy works best for ${ }^{57} \mathrm{Fe}$. Incoherent QENS works best for ${ }^{1} \mathrm{H}$ and, moderately well, for, e.g. ${ }^{2} \mathrm{H},{ }^{7} \mathrm{Li}, \mathrm{Na}, \mathrm{Ag}$ and $\mathrm{Cl}$. This is a restriction, but not so severe because protons are nearly ubiquitous and occur in all biological and organic matter, in metal hydrides and in proton conducting oxides. The other restriction is the restricted energy resolution of the neutron spectrometers: again, protons are favorable because they are, due to their small size, particular mobile and cause well measurable QENS line broadenings at easily accessible temperatures. Since the wavelength and the kinetic energy of cold and thermal neutrons is comparable to interatomic distances and excitation energies in condensed matter, respectively, the diffusion process can be studied on atomic scales of space and time simultaneously, and this feature is unique. For X-rays with wavelengths of a few $\AA$ the energies are in the $\mathrm{keV}$ range, and for Raman or Brillouin light scattering the energy fits to excitation energies in condensed matter, but the wavelength is far too large. The elucidation of the atomic diffusion and rotation mechanism is the strength of QENS rather than the determination of rotational and translational diffusion coefficients, which is also possible.

\section{Neutron scattering basics}

Neutrons, being constituents of nuclei, are liberated in nuclear reactions of neutron-rich nuclei. In research reactors fission of ${ }^{235} \mathrm{U}$ is utilized, in neutron spalla- 
tion sources neutrons appear when heavy nuclei like lead are bombarded by $\mathrm{GeV}$-protons. The mass of the neutron is very similar to the proton mass, the electric charge is zero, the spin is $\frac{1}{2}$, the magnetic moment $\mu_{n}=-1.913$ nuclear magnetons and the life time $10.6 \mathrm{~min}$. In the instant of generation neutrons have high kinetic energies (some $\mathrm{MeV}$ [48]). For application in neutron scattering these neutrons are slowed down and finally thermalized in so-called moderators. After moderation (thermal moderator water, preferably heavy water, cold moderator liquid $\mathrm{H}_{2}$ or $\mathrm{D}_{2}$ ) the temperature of the "neutron gas" approximately equals the moderator temperature, with a Maxwellian velocity distribution. A typical thermal neutron has an energy of $25 \mathrm{meV}$, a mean square velocity of $2200 \mathrm{~ms}^{-1}$ and, via de Broglie, a wavelength of $1.8 \AA$. A typical cold neutron has an energy of $1.9 \mathrm{meV}$, a mean square velocity of $600 \mathrm{~ms}^{-1}$ and a wavelength of $6.6 \AA$. In condensed matter, neutrons as neutral particles do not interact with the charge density of electrons (but as magnetic particles they do interact with spin density) but they interact with the nuclei and can be scattered or absorbed. Both processes are characterized by cross-sections which are listed in tables in many neutron scattering books and in the internet e.g. at http://www.ncnr.nist.gov/resources/ n-lengths/. Some nuclei, like ${ }^{3} \mathrm{He},{ }^{10} \mathrm{~B},{ }^{6} \mathrm{Li},{ }^{113} \mathrm{Cd}$ have a very high neutron absorption cross section and are used either for neutron detection or for shielding. On samples with these nuclei scattering experiments can hardly be performed. The neutron scattering at a nucleus is described by a scattering length $b$ which is, in contrast to X-ray scattering, not a function of the scattering angle because the neutron wavelength is much larger than the size of the nucleus. The irregularity of the nuclear scattering length with the chemical element number $\mathrm{Z}$ and its relative large value for light elements allows, other than X-ray scattering, elements adjacent in the periodic table to be distinguished and plays an important role for hydrogen compounds and for the investigation of light elements in the presence of heavy elements.

There are many atoms/elements where the nuclear species consist of several isotopes with scattering lengths $b_{1}, b_{2}, \ldots$, and corresponding abundances $c_{1}$, $c_{2}, \ldots$ The average of the scattering length is given by

$$
\langle b\rangle=\sum_{k} c_{k} b_{k}
$$

The corresponding cross-section is called the coherent neutron scattering cross-section

$$
\sigma_{\text {coh }}=4 \pi\langle b\rangle^{2}
$$

Since the scattering lengths of the isotopes are different, there appears socalled isotope incoherent scattering defined by the incoherent neutron scattering cross-section

$$
\sigma_{\text {inc }}=4 \pi\left(\left\langle b^{2}\right\rangle-\langle b\rangle^{2}\right)
$$

where the average of the squared scattering lengths is given by 


$$
\left\langle b^{2}\right\rangle=\sum_{k} c_{k} b_{k}^{2}
$$

The cross-section $\sigma_{\text {inc }}$ describes the square fluctuation of $\mathrm{b}$ due to isotope disorder. The sum $\sigma=\sigma_{\text {inc }}+\sigma_{\text {coh }}$ is called the total (bound) scattering crosssection. Coherent interference effects, which contain all the information on the cooperative properties of the scattering system, arise only from the average scattering potential experienced by the neutron, i.e. the result is the same as if all the nuclei were assigned the same scattering length $\langle b\rangle$. Deviations from this average potential are randomly distributed and produce a cross-section which, for elastic scattering, is isotropic. The incoherent cross-section can only be zero for single isotopes with zero nuclear spin. Some examples with their natural abundances given in parenthesis are ${ }^{12} \mathrm{C}(98.9 \%),{ }^{16} \mathrm{O}(99,8),{ }^{17} \mathrm{~F}(100 \%)$, ${ }^{27} \mathrm{Al}(100 \%),{ }^{28} \mathrm{Si}(92,2),{ }^{93} \mathrm{Nb}(100 \%)$. Simultaneously, these elements have very low neutron absorption cross-sections. Therefore graphite, diamond, aluminium, corundum $\left(\mathrm{Al}_{2} \mathrm{O}_{3}\right)$ and niobium are favorite construction materials in the sample periphery (e.g. furnaces, cryostats, pressure cells).

For nuclei with nuclear spin $I \neq 0$ there appears an additional scattering contribution, called spin incoherent scattering, if the scattering depends on the relative orientation of neutron and nuclear spin. Let us consider a single-isotope specimen with the nuclear spin I. The interaction of the spin $\frac{1}{2}$ neutron with the nuclear spins produces two compound nuclear states with total spins parallel $I$ $+\frac{1}{2}$ and antiparallel $I-\frac{1}{2}$ with different scattering lengths $b^{+}$and $b^{-}$, respectively. The corresponding average scattering lengths are then given by

$$
\begin{aligned}
& \langle b\rangle=\left(\frac{I+1}{2 I+1}\right) b^{+}+\left(\frac{I}{2 I+1}\right) b^{-} \\
& \left\langle b^{2}\right\rangle=\left(\frac{I+1}{2 I+1}\right)\left(b^{+}\right)^{2}+\left(\frac{I}{2 I+1}\right)\left(b^{-}\right)^{2}
\end{aligned}
$$

For the hydrogen/proton with $I=\frac{1}{2}, \mathrm{~b}^{+}=10.8 \mathrm{fm}$ and $\mathrm{b}^{-}=-47.4 \mathrm{fm}$ the coherent cross-section $\sigma_{\text {coh }}=4 \pi\langle b\rangle^{2}=1.76$ barn $=1.76 \cdot 10^{-28} \mathrm{~m}^{2}$ (tabulated value) is rather small and the incoherent cross-section $\sigma_{\text {inc }}=4 \pi\left(\left\langle b^{2}\right\rangle-\langle b\rangle^{2}\right)=$ 80.26 barn (tabulated value) is extraordinary large. For deuterium $\sigma_{\text {coh }}=$ 5.59 barn and $\sigma_{\text {inc }}=2.09$ barn are of the same order of magnitude. The spin dependent scattering can be utilized to separate coherent and spin-incoherent quasielastic neutron scattering by means of polarized neutrons, i.e. with a polarized incident neutron beam and with polarization analysis of the scattered neutrons [6]. We consider an incident beam with spin-up polarization. Coherent QENS does not flip the polarization, whereas in spin-incoherent QENS one third of the scattering intensity is non-spinflip and two thirds are spinflip scattering:

$$
\left(\frac{d \sigma}{d \Omega}\right)^{\uparrow \uparrow}=\left(\frac{d \sigma}{d \Omega}\right)_{\text {coh }}+\frac{1}{3}\left(\frac{d \sigma}{d \Omega}\right)_{\text {spin-inc }}
$$




$$
\left(\frac{d \sigma}{d \Omega}\right)^{\uparrow \downarrow}=\frac{2}{3}\left(\frac{d \sigma}{d \Omega}\right)_{\text {spin-inc }}
$$

hence:

$$
\begin{aligned}
& \left(\frac{d \sigma}{d \Omega}\right)_{\text {coh }}=\left(\frac{d \sigma}{d \Omega}\right)^{\uparrow \uparrow}-\frac{1}{2}\left(\frac{d \sigma}{d \Omega}\right)^{\uparrow \downarrow} \\
& \left(\frac{d \sigma}{d \Omega}\right)_{\text {spin-inc }}=\frac{3}{2} \cdot\left(\frac{d \sigma}{d \Omega}\right)^{\uparrow \downarrow}
\end{aligned}
$$

There are not many examples of quasielastic neutron scattering experiments in which the separation between coherent and incoherent contributions has been performed. But with the progress in instrumentation the situation will change in the future. Using polarization not only strong Bragg peaks (with often significant tails under the QENS spectrum) can be eliminated, but also the always used incoherent approximation of QENS spectra gets really true. Furthermore it helps enormously to determine the EISF and the Debye-Waller factor [7] (see 3.1). Currently such measurements have to be performed on a triple axis spectrometer, which is much time-consuming because of the point-by-point measurement (fixed $\mathrm{E}_{i}$ and $\mathrm{E}_{f}$ for one point) in the large Q- $\omega$ space.

We now come back to unpolarized neutrons. Let us consider a system with only one species and let us consider only the QENS signal from this mobile species. Then the intensity of the scattered neutrons is proportional to the double differential cross-section

$$
\frac{d^{2} \sigma}{d \Omega d \omega}=\frac{k_{f}}{k_{i}}\left\{\frac{\sigma_{\mathrm{coh}}}{4 \pi} S_{\mathrm{coh}}(Q, \omega)+\frac{\sigma_{\mathrm{inc}}}{4 \pi} S_{\mathrm{inc}}(Q, \omega)\right\}
$$

where $k_{i}$ is the wave number of the incident neutrons and $k_{f}$ is the wave number of the scattered neutrons.

Strictly speaking, the scattering process is a quantum mechanical transition and has to be treated in terms of wave functions, transition probabilities, timedependent perturbation theory etc. and, eventually, ensemble averages. On the other hand, classical particle diffusion in condensed matter is treated in terms of statistical physics. The interface between these quite different types of descriptions is given by thermal averages which can be expressed in terms of so-called van Hove correlation functions, $G(\mathbf{r}, t)$ and $G_{s}(\mathbf{r}, t)$. The correlation function $G(\mathbf{r}, t)$ - $d \mathbf{r}$ is the conditional probability that, given a particle was at time $t=0$ at the origin $\mathbf{r}=0$, any particle is found at time $\mathrm{t}$ at the position $\mathbf{r}$ in a volume element $\mathrm{d} \mathbf{r}$. The self-correlation function $G_{s}(\mathbf{r}, t) \cdot \mathrm{d} \mathbf{r}$ is the conditional probability that, given a particle was at time $\mathrm{t}=0$ at the origin $\mathbf{r}=0$, the same particle is found at time $\mathrm{t}$ at the position $\mathbf{r}$ in a volume element $\mathrm{d} \mathbf{r}$.

The Fourier transformation of $\mathrm{G}(\mathbf{r}, \mathrm{t})$ with respect to space yields the socalled intermediate scattering function $\mathrm{I}(\mathbf{Q}, \mathrm{t})$, and a subsequent temporal Fourier transformation yields the dynamical scattering function 


$$
S(\mathbf{Q}, \omega)=(2 \pi \hbar)^{-1} \int_{-\infty}^{+\infty} \int^{i(\mathbf{Q} \mathbf{r}-\omega t)} G(\mathbf{r}, t) d \mathbf{r} d t
$$

The analogous treatment of the self-correlation function yields, via the selfpart of the intermediate scattering function $\mathrm{I}_{s}(\mathbf{Q}, \mathrm{t})$, the incoherent scattering function:

$$
S_{\text {inc }}(\mathbf{Q}, \omega)=(2 \pi \hbar)^{-1} \int_{-\infty}^{+\infty} \int^{i(\mathbf{Q}-\omega t)} G_{s}(\mathbf{r}, t) d \mathbf{r} d t
$$

With respect to diffusion, $\mathbf{S}(\mathbf{Q}, \omega)$ gives access to collective diffusion and $\mathrm{S}_{\text {inc }}(\mathbf{Q}, \omega)$ gives access to self-diffusion. But at very low concentration of scattering species the situation is special: Coherent scattering comprises a self-term and a distinct term, because 'any scatterer' could be the same scatterer (self-term) or a different scatterer. At very low concentration the probability to find a different scatterer at $(\mathbf{r}, \mathbf{t})$ is negligible, and only the self-terms remains which thus yields access to self-diffusion.

\section{Applications of the QENS method}

The method of QENS enables investigations of dynamical processes on $10^{-13}$ $10^{-7} \mathrm{~s}$ time-scale. The QENS signal is due to diffusive (stochastic) atomic or molecular motions.

Concerning the different types of stochastic motions which can be studied by the QENS method we have to differentiate between localized and long-range diffusion. To make this statement clear we present in Fig. 1 the corresponding intermediate scattering function $\mathrm{I}_{s}(\mathrm{Q}, \mathrm{t})$ and the scattering function $\mathrm{S}_{\mathrm{inc}}(\mathrm{Q}, \omega)$ for a constant $Q$ value.

In the case of long-range self-diffusion $I_{s}(Q, t)$ decays to zero for $t \rightarrow \infty$, while for localized motions (like rotations or diffusion inside a confinement) this function approaches a finite value for $t \rightarrow \infty$. The corresponding scattering function $S_{\text {inc }}(Q, \omega)$ consists of a pure Lorentzian in the case of long-range diffusion (in the simplest case) and in the case of a localized motion $S_{\text {inc }}(Q, \omega)$ is composed of a $\delta(\hbar \omega)$-peak and one ore several Lorentzian.

\subsection{The elastic incoherent structure factor}

One of the key parameters in QENS is the so-called elastic incoherent structure factor. In general the self-part of the van Hove correlation function can be written as:

$$
G_{s}(\mathbf{r}, t)=G_{s}(\mathbf{r}, \infty)+\widetilde{G}_{s}(\mathbf{r}, t)
$$

The first term in Eq. 16 is the value of the correlation function at very long times, i.e. for $t \rightarrow \infty$. If the particle explores a volume that is large in comparison 


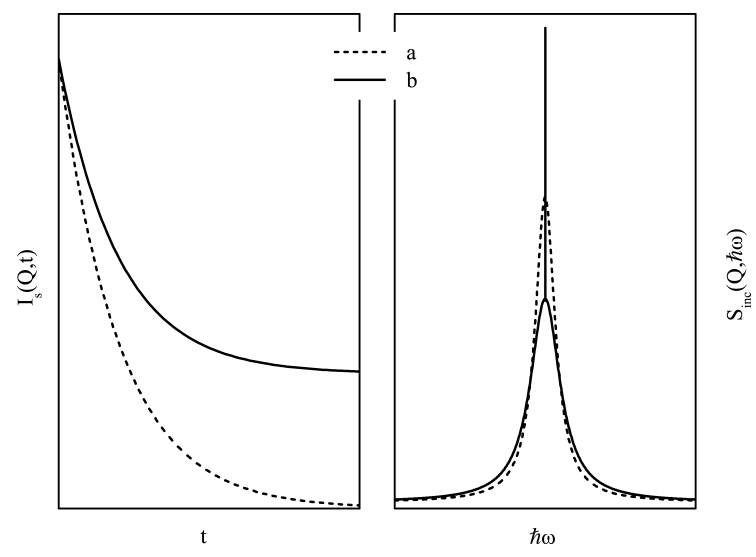

Fig. 1. Self-part of the intermediate scattering function (left) and incoherent scattering function (right) for long-range diffusion (a, dashed line) and for a localized dynamical process (b, solid line).

to the interatomic distances of the system, then $G_{s}(\mathbf{r}, \infty)=0$; however if the particle's motion is restricted to some finite volume, $G_{s}(\mathbf{r}, \infty)$ has a finite value. Performing two Fourier transforms (with respect to time and space, respectively) we get the incoherent scattering function, introduced in the preceding section. This incoherent scattering function also consists of two terms:

$$
S_{\text {inc }}(\mathbf{Q}, \omega)=S_{\text {inc }}^{\text {el }}(\mathbf{Q})+S_{\text {inc }}^{\text {inel }}(\mathbf{Q}, \omega)
$$

The first term is a purely elastic contribution, while the second one is the inelastic component. The elastic line can be considered as resulting from the diffraction of the neutron on the infinite time distribution in space of the nuclei belonging to the scattering species over a finite volume by its motion. In this sense, one can get information on the structure from incoherent scattering. The concept of the elastic incoherent structure factor (EISF) was introduced by R. Lechner in 1971 (see [4] and corresponding references therein). The EISF can be determined experimentally if a discrimination between the elastic and nonelastic components is possible. In these cases the EISF is given by the following relation:

$$
E I S F=\frac{I^{\mathrm{el}}}{I^{\mathrm{el}}+I^{\text {inel }}}
$$

where $I^{\mathrm{el}}$ denotes the integrated elastic intensity and $I^{\text {inel }}$ is the inelastic intensity. In this definition, the EISF contains all the dynamical processes of the scattering particle under investigation. Usually only one type of motion can be investigated with one experiment, i.e. with one given resolution of the experimental setup used. In this case one can apply the EISF concept on the elastic component of this specific type of motion. The expression in Eq. 18 has to be modified slightly, 
because the inelastic component consists now of the quasielastic contribution of the specific motion. In analogy to Eq. 18 the EISF is given by

$$
E I S F=\frac{I^{\mathrm{el}}}{I^{\mathrm{el}}+I^{\mathrm{qel}}}
$$

where $I^{\mathrm{qel}}$ denotes the integrated quasielastic intensity.

Finally we note that the presence of an elastic scattering component is a hint for a localized motion, provided that this intensity is not the result of scattering from a host lattice (one may think for example in terms of so-called inclusion compounds for which the host builds up a skeleton in which guest molecules can exist).

\subsection{Self-diffusion}

As the most simple application of the formalism presented in Section 2, we consider long-range self-diffusion, i.e. small-Q incoherent neutron scattering. The self-correlation function obeys Fickss second law of diffusion:

$$
\frac{\partial}{\partial t} G_{\mathrm{s}}(r, t)=D_{\mathrm{s}} \nabla^{2} G_{\mathrm{s}}(r, t)
$$

with the initial condition $\mathrm{G}_{s}(\mathrm{r}, \mathrm{t}=0)=\delta(\mathrm{r})$. Thus we treat the diffusion process as if all diffusing particles start at the origin at time zero; this leads to the correct result because of temporal and spatial invariance. The solution is

$$
G_{s}(r, t)=\left(4 \pi D_{s}|t|\right)^{-3 / 2} \exp \left(-\frac{r^{2}}{4 D_{s}|t|}\right)
$$

Spatial Fourier transformation yields the self-part of the intermediate scattering function

$$
I_{s}(Q, t)=\exp \left(-Q^{2} D_{s} t\right)
$$

and the subsequent Fourier transformation with respect to time yields the incoherent scattering function

$$
S_{\text {inc }}(Q, \omega)=\frac{1}{\pi} \frac{\hbar D_{s} Q^{2}}{\left(\hbar D_{s} Q^{2}\right)^{2}+(\hbar \omega)^{2}}
$$

which is a Lorentzian function with the half-width at half maximum (HWHM)

$$
\Gamma=\hbar D_{s} Q^{2}
$$

At sufficiently small $\mathrm{Q}$ this so-called $\mathrm{Q}^{2}$-law is generally valid irrespective of the details of the diffusion process, which can be elucidated from measurements at finite or larger Q, but any long-range diffusion model converges to the $\mathrm{Q}^{2}$ law at small $\mathrm{Q}$.

\subsection{Jump diffusion}

The diffusion on the microscopic scale can be successfully described by socalled jump diffusion models. These models assume that diffusion takes place as 
successive jumps. Parameters are D, the diffusion coefficient, $\tau$, the so-called residence time and the mean-jump length, from which only two are independent. Different models have been developed for different jump length distributions. We start with the one proposed by Chudley and Elliott [8]. In this model the jump length $r$ is fixed and one gets for the half-width at half maximum (HWHM) the following expression (after orientational averaging):

$$
\Gamma(Q)=\frac{\hbar}{\tau}\left(1-\frac{\sin (Q r)}{Q r}\right)
$$

$\tau$ denotes here the time the particles spends at a new position before it jumps again to a new position.

The model proposed by Hall and Ross [9] deals with a Gaussian distribution of jump-length

$$
\rho(r)=\frac{2 r^{2}}{r_{0}^{3} \sqrt{2 \pi}} \cdot e^{-r^{2} / 2 r_{0}^{2}}
$$

with the mean-square-displacement $(m s d)\left\langle r^{2}\right\rangle=\int_{0}^{\infty} r^{2} \rho(r) d r=3 r_{0}^{2}$. The HWHM for this model reads:

$$
\Gamma(Q)=\frac{\hbar}{\tau}\left(1-e^{-Q^{2} r_{0}^{2} / 6}\right)
$$

Finally, Singwi and Sjölander [10] proposed an exponential distribution of jump lengths:

$$
\rho(r)=\frac{r}{r_{0}^{2}} \cdot e^{-r / r_{0}}
$$

This distribution gives the following QENS line width (as function of Q):

$$
\Gamma(Q)=\frac{\hbar}{\tau} \frac{Q^{2} r_{0}^{2}}{1+Q^{2} r_{0}^{2}}
$$

with the msd given by $\left\langle r^{2}\right\rangle=6 r_{0}^{2}$.

Note that Eqs. 25, 27 and 29 yield in the small Q limit the generally valid $\mathrm{Q}^{2}$-law (long-range diffusion)

$$
\Gamma(Q)=\hbar D Q^{2}
$$

with the diffusion coefficient $D=\frac{\left\langle r^{2}\right\rangle}{6 \tau}$. In the large Q limit all models yield $\hbar /$ $\tau$, where $\tau$ represents the time between two successive jumps.

\subsection{Rotational dynamics}

Now we consider the isotropic rotational diffusion (continuous rotational diffusion on the surface of a sphere). The idea behind this model is that the reorientation of the molecule is due to small-angle random rotations. The orientation of a particle is described by an orientation vector $\Omega=(\phi, \theta)$. For the orientation probability $P(\Omega, t)$ of an orientation $\Omega$ at time $t$ it can be written, in analogy to Fick's law: 


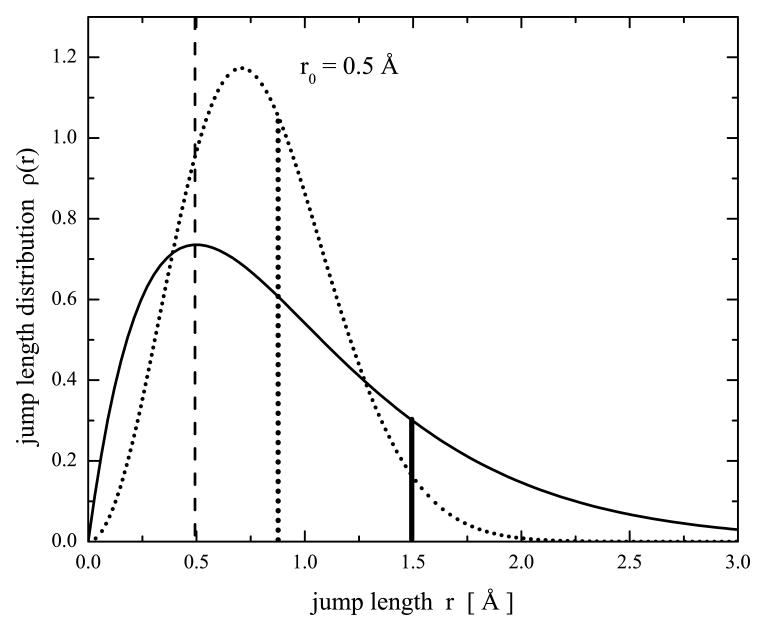

Fig. 2. The distribution of jump lengths according to the model proposed by Hall and Ross (dotted line) and by Singwi and Sjölander (solid line). For both distributions the same $r_{0}=$ $0.5 \AA$ has been chosen. The vertical lines denote $r_{0}$ (dashed line), the mean jump length for the Hall-Ross model (dotted line) and the mean jump length for the model of Singwi and Sjölander (solid line).

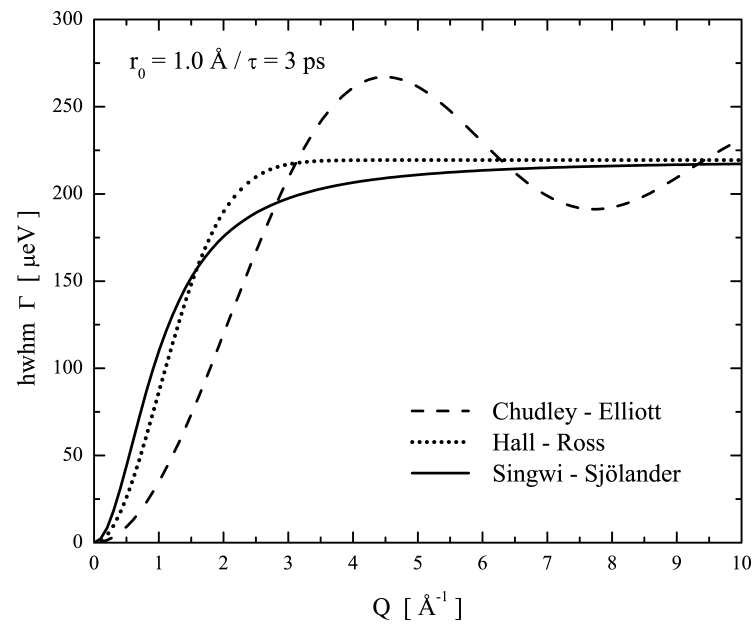

Fig. 3. HWHM as a function of Q for the three different models; in all cases $r_{0}$ was set to $1.0 \AA$ and $\tau$ to 3 ps. According to Eq. 30 the diffusion coefficient is different for all the models, but $r_{0}$ is kept constant.

$$
\frac{\partial}{\partial_{t}} P(\vec{\Omega}, t)=D_{R} \nabla^{2} P(\vec{\Omega}, t)
$$


The solution of Eq. 31 then reads (taking into account appropriate boundary conditions)

$$
I_{s}^{R}(Q, t)=\sum_{l=0}^{\infty}(2 l+1) j_{l}^{2}(Q R) e^{-D_{R} l(l+1) t}
$$

and in energy-domain the corresponding scattering function is given by

$$
S_{s}^{R}(Q, t)=j_{0}^{2}(Q R) \delta(\hbar \omega)+\sum_{l=1}^{\infty}(2 l+1) j_{l}^{2}(Q R) \frac{1}{\pi} \frac{\hbar D_{R} l(l+1)}{\left(\hbar D_{R} l(l+1)\right)^{2}+(\hbar \omega)^{2}}
$$

where $j_{l}(x)$ denotes spherical Bessel functions of the order $l$. The corresponding EISF for this model is given by $j_{0}{ }^{2}(Q R)$, where $R$ is the rotational radius, e.g. the distance of the rotating atoms from the center of mass of the molecule.

\subsection{Reorientations}

The simplest form of a reorientational type of motion consists of jumps between two equivalent sites (dumb-bell, particle jumps back and forth between iso-energetic sites); for a complete derivation of the scattering function see [3]). The scattering function for this motion is given by

$$
S_{s}(Q, \omega)=A_{0}(Q) \delta(\hbar \omega)+\left(1-A_{0}(Q)\right) \frac{1}{\pi} \frac{2 \hbar / \tau}{(2 \hbar / \tau)^{2}+(\hbar \omega)^{2}}
$$

In Eq. $34 \tau$ denotes the time between two successive jumps. $A_{0}(Q)=\frac{1}{2}$ $\left(1+j_{0}(Q d)\right)$ represents the EISF with $d$ the jump distance between the two sites. This simple model can be extended to the case of either two energetically inequivalent sites or to jumps among $N$ sites. For $N$-equivalent sites located on a circle with radius $R$, the incoherent scattering function reads:

$$
S_{s}(Q, \omega)=A_{0}(Q) \delta(\hbar \omega)+\sum_{j=1}^{N} A_{j}(Q) \frac{1}{\pi} \frac{\hbar / \tau_{j}}{\left(\hbar / \tau_{j}\right)^{2}+(\hbar \omega)^{2}}
$$

with

$$
\begin{aligned}
& A_{k}(Q)=\frac{1}{N} \sum_{n=1}^{N} j_{0}\left(Q r_{n}\right) \cos (2 l \pi n / N) \\
& r_{n}=2 R \sin (\pi n / N) \\
& \tau_{j}^{-1}=\frac{2}{\tau} \sin ^{2}(\pi j / N) .
\end{aligned}
$$

Note that $\tau_{0}^{-1}=0$ means pure elastic scattering.

\subsection{Data analysis - general aspects}

The experimentally obtained QENS spectra are normally not well structured. For this reason data analysis is often complicated and not straight forward. Neverthe- 


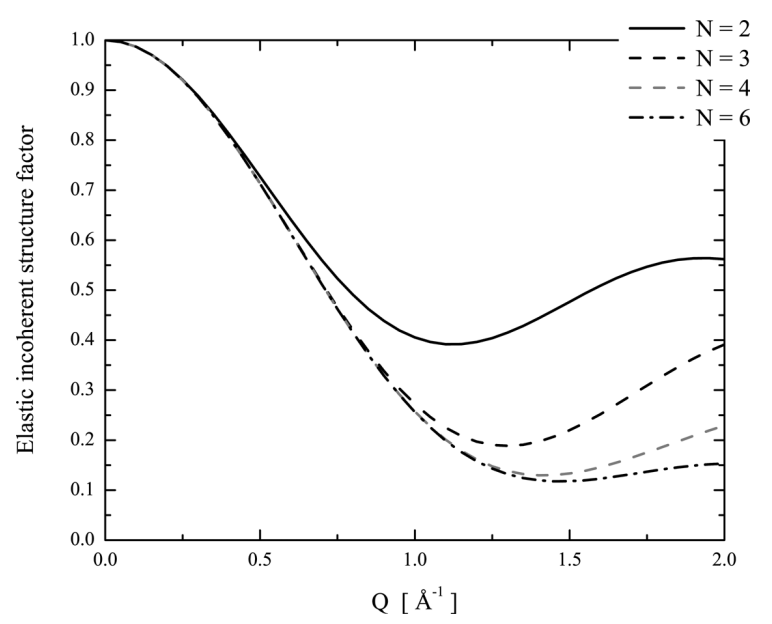

Fig. 4. EISF $A_{0}(Q)$ for $\mathrm{R}=2 \AA$ and different values of $\mathrm{N}$.

less fitting the data in Q- $\omega$ space with one or two Lorentzian functions or with one Lorentzian and one elastic line is in many cases a good starting point. If it is possible to fit the data with an elastic line and a Lorentzian-like contribution the EISF for this dynamical process can be determined in first approximation. The information given by the EISF may further result in a first idea about a model describing the process under investigation. Sometimes the model can only be written down in the time domain, i.e. for example the Fourier transform from $\mathrm{I}(\mathrm{Q}, \mathrm{t})$ to $\mathrm{S}(\mathrm{Q}, \omega)$ can't be done in an analytical way. In these cases it may be useful to transform the measured data into time domain. Example for this approach are models involving so-called stretched exponentials given by

$$
I(Q, t) \propto \exp \left(-(t / \tau)^{\beta}\right)
$$

From time to time a further step is needed in order to describe the experimentally determined data with a correct model that reflects all observations. This further step consists of fitting all the data in a simultaneous manner. Here simultaneous means that all available Q-groups are fitted with one model at the same time, instead of fitting each spectrum individually, and thus reducing the number of fit parameters needed to describe the data.

As already mentioned the EISF is the key parameter accessible in QENS investigations. The determination of this parameter may however be difficult or at least not straight forward. One example for this statement is the presence of a host material in the sample. Very often the host material shows up only with elastic contributions and thus using Eq. 18 will result in an incorrect EISF. The dynamical structure factor in this case can be written in the following form

$$
S(Q, \omega)=A \cdot \delta(E)+(1-A) \cdot S_{\text {model }}(Q, \omega)
$$

with $S_{\text {model }}(Q, \omega)=a \cdot \delta(E)+(1-a) \cdot L(\Gamma, E)$, and so EISF $=$ a. 
Another complication may arise when two or even several dynamical processes are coupled. The simplest example is the combination of a rotational diffusion with a long-range diffusion. In this example the $\mathrm{S}(\mathrm{Q}, \omega)$ is composed of Lorentzians exclusively, i.e. the $\delta$-term has broadened. Nevertheless the prefactor of the first Lorentzian is just given by the EISF.

In the case of very complex systems the QENS data interpretation is substantially supported by molecular dynamics simulations. From the experimental point of view the data accuracy has to be good enough in order to distinguish between different possible models.

\section{Neutron instrumentation}

In this chapter QENS instruments are described, such that new users might be assisted to plan their experiments. There are many books and papers, which describes this topic more fundamentally and detailed [11].

Neutron scattering instruments are designed individually to be optimized for certain types of instruments. However there are some typical instrument types and components, those for QENS experiments are presented below. Important materials while constructing instruments are:

- Aluminium - highly transparent for neutrons, cheap

- Stainless steel - good mechanical properties, non-magnetic

$-{ }^{113} \mathrm{Cd},{ }^{10} \mathrm{~B},{ }^{155} \mathrm{Gd},{ }^{157} \mathrm{Gd},{ }^{6} \mathrm{Li}$ - neutron absorbers

- Polyethylene, concrete - moderators

- Lead - $\gamma$-shielding

\section{Neutron guides}

The instruments are usually several meters away from the source, therefore an efficient system is needed to transport neutrons with minimum loss of intensity. The major problem for neutrons is the very low critical angle, below which neutrons have total reflection. State-of-the-art device is the supermirror [12]. It consists of artificial multilayers, with smoothly varying layer thicknesses, so the region of total reflection is extended by quasi-overlapping Bragg reflections. An important measure is the ratio of this effective critical angle and the critical angle of $\mathrm{Ni}$, often denoted by $m$. On the other hand, large $m$ results in higher beam divergency, so the intensity increases while at the same time the Q-resolution decreases. The critical angle is proportional to the neutron wavelength. Near to the source the guides are usually curved, and so unwanted high energy neutrons (for which the critical angle is low) are not transported to the instruments.

\section{Choppers}

Choppers are rotating devices which "chop" the neutron beam, i.e. neutrons can pass the chopper only for certain times, periodically. They are mainly used to 
select neutrons having a certain energy/velocity. Furthermore they are used for background reduction and to avoid frame overlap [49]. A disc-chopper is a disc rotating around its axis, which is parallel to the neutron beam. The disc is coated with an absorbing material, except the window(s). The windows have at least the same width as the neutron beam; furthermore the disc radius is limited due to the high rotation speed (some thousand rotation per minute), therefore it is open for relatively long time. If short pulses are required a so-called Fermi Chopper can be used. In this case a slit package (repeated layers of absorbing and non-absorbing materials with a total thickness of some $\mathrm{cm}$ ) is used. Its rotation axis is perpendicular to the neutron beam. Neutrons with a certain energy can be selected using at least two choppers based on their time of flight. This energy can be varied by changing the phase between the choppers.

\section{Monochromators}

Alternatively neutron energies can be selected using Bragg reflection of a crystal. Monochromators are neutron optical devices built up from one or several crystals. If the guide - monochromator - sample angle is changed (and the monochromator is rotated correspondingly) the energy can be varied. The monochromator crystal is chosen based on its d-spacing, mosaicity [50], reflectivity. Pyrolythic graphite, Silicon, Germanium, Copper are often used. Monochromators are usually curved either in one or two directions to focus the beam on the sample and thus to increase intensity. Focussing in the scattering plane also effects the energy resolution. The same device is called analyzer, if it is placed behind the sample, and so used to analyze the neutron's energy after the scattering event.

\section{Filters}

Neutron transmission depends on the neutron energy. Below a 'cut-off' neutron wavelength the transmission may decrease dramatically due to the onset of Bragg scattering in the filter (see Eq.1, $\lambda_{\text {cut-off }}=2 d$ ). This effect is used to filter the neutron beam, for example to avoid higher order scattering on monochromators. Polycrystalline Beryllium is often used, its 'cut-off' energy is $5.1 \mathrm{meV}$ (4 ̊).

\section{Detectors and monitors}

Quasielastic and also inelastic neutron scattering intensity is often weak, therefore detection efficiency has high priority. Furthermore the Q resolution is not critical. Therefore mostly tubes filled with ${ }^{3} \mathrm{He}$ at a pressure of some bar are used as detectors with efficiency close to $100 \%$. When a neutron hits a ${ }^{3} \mathrm{He}$ nucleus a proton and a triton with $764 \mathrm{keV}$ kinetic energy are produced and thus the surrounding gas gets ionized. This "cloud" is amplified by high voltage (proportional counter) and the created electrons will than be detected as charge pulses. These detectors also measure $\gamma$-rays [51], but $\gamma$-radiation can be discriminated effectively. The detector systems deliver usually the neutron counts as a 
function of time, some times even as a function of position along a long tube (so-called position sensitive detectors (PSD)).

Monitors are low efficiency detectors, and usually placed directly in front of the sample. Their count rate is proportional to the neutron intensity hitting the sample, and therefore they are used to normalize the data with respect to the incoming flux. The neutron detection is based either on ${ }^{235} \mathrm{U}$ fission or on absorption by ${ }^{3} \mathrm{He}$.

\section{Collimators}

The neutron beam has a finite divergence [52]. This can be reduced mechanically by collimators (or by pin holes). A so-called Soller collimator consists of thin absorbing lamellas with certain length and distance between them, filling the beam cross section. A radial collimator (absorber lamellas with certain length and angle between them) can be placed around the sample. This is used to avoid those neutrons reaching the detectors, which are scattered in the sample environment. The radial collimator is often oscillating in order to avoid shadows (due to the lamella material) at certain scattering angles.

\section{Flight paths}

Ideally, longer flight paths are under vacuum to avoid air-scattering. For shorter distances this might be unpractical due to rather robust vacuum vessels, i.e. a large amount of material in the beam. If small angle scattering is tolerable, Argon, filled in containers with thin Al windows, is a good choice.

\section{Shielding}

The instruments are shielded for two purposes: to keep radiation outside the instrument low, and to reduce background scattering in the detector. A shielding might be constructed as a sandwich, for example polyethylene to slow down thermal neutrons followed by so-called Borplastic [53] to stop them. In addition neutrons may produce $\gamma$-rays, for example when they are absorbed by Cadmium or Gadolinium. Therefore $\gamma$-shielding might be necessary as well, but mainly to reduce the scattering outside the instrument. Furthermore most instruments have a so-called beamstop behind the sample to catch the high intensity of the transmitted beam.

\section{Sample environment}

Exploring new physics, chemistry, biology, etc. means new materials and extreme conditions. Extreme sample conditions can be provided by state of the art sample environment devices. A strong advantage of neutron scattering is the large variety of those, due to the deep penetration of neutrons into matter. It is possible to apply magnetic fields (up to $17.5 \mathrm{~T}$ ), pressure (up to $25 \mathrm{GPa}$ ) and extreme temperature (ranging from $15 \mathrm{mK}$ up to $2273 \mathrm{~K}$ ) using standard equipments; however the limits differ from device to device. With restrictions these 
can also be combined. Furthermore magnetic or electric levitation, shear and stress devices, electric fields, etc. are also available.

\subsection{Time-of-flight spectrometers}

Time-of-flight spectrometers measure intensities proportional to $\mathrm{S}(\mathrm{Q}, \omega)$. The energy transfer is measured by the time-of-flight technique. The momentum transfer Q is not directly measured, but calculated from the scattering angle and the energy transfer. The usual QENS signal covers a continuous range within the Q$\omega$ space, therefore simultaneous measurement of these points is aimed. The incident neutron beam has a well defined direction, and the scattered neutrons are detected at a number of scattering angles. There are two possible instrument configurations: either a monochromatic neutron beam hits the sample and the scattered neutron energies are measured (mostly by time-of-flight), or the energy of the incoming neutrons covers a defined but broad range ("white" incident beam), while only part of the scattered neutrons, which have a fixed energy are detected (mostly selected by analyzers). The former type of instruments are the 'direct geometry'-, whereas the later ones are the 'indirect geometry' spectrometers. The direct geometry spectrometers monochromize the neutron beam by a chopper system or by a monochromator. Since the energy of the scattered neutrons are best measured based on their time-of-flight, even the crystal tof-spectrometers have at least one chopper to produce periodic pulses. The energy resolution varies from some $\mu \mathrm{eV}$ to some meV by changing the incident energy. Inverted time-of-flight spectrometers use a chopper system to select neutrons in a defined but broad energy range. The scattered neutrons are selected by analyzers, so only neutrons with a certain final energy reach the detectors. Such instruments are suitable for QENS measurement because of the restricted energy window paired with higher flux, however they do not have the flexibility of the direct tof-spectrometers.

\subsection{Backscattering spectroscopy}

Backscattering spectrometers can also be build either in direct- or indirect geometry. In contrast to time-of-flight spectrometers, the energy transfer is measured by using analyzers. The best energy resolution of a monochromator is in backscattering position, i.e. $\theta=90^{\circ}$. Traditional backscattering spectrometers use monochromators and analyzers in close to backscattering position, reaching an energy resolution even below $1 \mu \mathrm{eV}$. The incident energy can be varied by moving the monochromator by a Doppler-drive or by changing the temperature and so the lattice spacing of the monochromator crystal. Unfortunately the energy window is quite limited on both cases. The so called fixed window scan [54] is often used to explore the temperature range, in which the dynamics of the sample has a time scale suitable for the instrument. Furthermore the temperature dependence of the Debye-Waller factor and so the temperature induced changes in the mean square displacement can be measured. The importance of energy resolution 
of the instrument and a critical discussions of the mean square displacement can be found in [13]. Nowadays also chopper TOF-spectrometers may provide 3-4 $\mu \mathrm{eV}$ resolution, however the covered $\mathrm{Q}$ range is significantly smaller and thus the details of the diffusion process cannot be investigated. The theory and the development of the backscattering technique can be followed on the web [14], furthermore the article of Frick et al. in this issue describes the actual status, new instruments and experimental results.

\subsection{Neutron spin echo}

In 1972 Ferenc Mezei invented a new principle to be able to measure even slower motions (for a detailed survey over this technique we refer to [15] and the article by Fouquet et al. in this issue): the neutron spin echo technique. Contrary to other QENS instruments, spin echo spectrometers directly measure $\mathrm{I}(\mathrm{Q}, \mathrm{t})$ rather than $\mathrm{S}(\mathrm{Q}, \omega)$, i.e. they measure directly the intermediate scattering function. The important difference is, that the better energy resolution does not cause further intensity reduction, because the measurement of the neutron energy is decoupled from neutron velocity. A neutron in a magnetic field (perpendicular to its magnetic moment) precesses around the field direction with the Larmor frequency $\omega=\gamma B$, where $\gamma=-2.92 \cdot 10^{7} \mathrm{HzT}^{-1}$ is the gyromagnetic ratio of the neutron. When the direction of the magnetic field changes, we have to distinguish between two limiting cases

- adiabatic, if the change is slow compared to the Larmor frequency, the neutron can follow

- non-adiabatic, opposite to the previous one.

The principle of this spectrometer is the following: a beam of polarized neutrons enters a magnetic field, and because of the precession the direction of its moment changes by an angle $\phi=\gamma \mathrm{B} l / \mathrm{v}$, where $l$ is the path length in the magnetic field, and $\mathrm{v}$ is the neutron velocity. After scattering at the sample, the neutrons precess through an opposite magnetic field. In case of elastic scattering, when the velocity did not change during the scattering process, the neutron spin turns back to the original position, and so the polarization is recovered (this is the so-called spin-echo; this technique was originally developed for NMR experiments), independent of its velocity. Contrary, in an inelastic scattering process the spin direction can be turned back if the magnetic field is different. Therefore by scanning the strength of the magnetic field before and after the sample the dynamics can be measured.

Reversing the magnetic field is difficult due to the fact that it implies a point at which the field gets zero; at this point the polarized beam will be (at least partially) depolarized. In practice so-called spin-flippers are used, which flip the polarization in an appropriate direction or reverse the sense of precession. This is of course an oversimplified description; more details are provided in $[16,15]$. 


\section{Experimental aspects}

Most neutron scattering facilities accept regular proposals only at some fixed deadlines, therefore both the proposal and the experiment should be planned very carefully. New users should contact the instrument scientist well before the proposal deadline and ask for advice. The instrument characteristics and contact persons can be found on the web site of the facilities. Additionally, details of the experiment, like sample environment devices, sample can etc., should be also discussed prior to the experiment. In the following some important issues are discussed.

\section{Which instrument to choose?}

The most important parameter is the instrument resolution, and secondly the required $Q$ range. If there is no idea about the timescale of the motion it is useful to start either at a chopper spectrometer, where the energy resolution can be varied from some meV to even some $\mu \mathrm{eV}$ (of course on the cost of intensity), or with a fixed window scan at a backscattering instrument (using a fixed resolution but changing the QENS broadening via temperature). Spin echo spectrometers cover a broad range of relaxation times (from some ps to some hundred ns); so they may be used for complex systems with a broad variety of dynamical processes. An instrument pathfinder, that may help to chose the appropriate instrument, is provided on the European Neutron Portal [17]. In Sec. 6 we describe the use of QENS to some dedicated systems ranging from liquid metals to complex biological samples aiming to describe a broad range of dynamical processes and timescales accessible via QENS.

\section{Sample size}

The beam size at the sample position is usually some $\mathrm{cm}$ both in horizontal and vertical directions, being more extended perpendicular to the scattering plane. Beam divergency, focussing, scattering process, etc. result in a nonuniform beam profile, having less intensity in the outer part. Generally, the sample size should comparable to the size of the beam. On the other hand, too large samples might worsen the energy resolution on time-of-flight instruments. When neutrons penetrate through the sample, part of the beam will be scattered or absorbed with a given probability. This fact results in an exponential reduction of the intensity of the transmitted neutron beam: $I=I_{0} \cdot e^{-\sigma N d}$, where $\sigma$ is the total cross section, $\mathrm{N}$ is the number density of scatterers, and $\mathrm{d}$ is the sample thickness in the direction of the neutron beam. Ideally the sample should scatter about $5-10 \%$ of the incoming neutrons. Otherwise the data have to be corrected additionally for multiple scattering (see [3] for further details). On some backscattering spectrometers the beam crosses the sample twice, therefore the transmission $\left(T=I / I_{0}\right)$ should be correspondingly higher. As an example, for pure water the optimal thickness is between $0.1-0.2 \mathrm{~mm}$. 


\section{Self-absorption}

The above mentioned exponential law is the reason for the so called self-absorption or self-attenuation effect. It is especially important, if one would like to subtract the exact amount of background. One can correct for that with Q and E dependent factors, which depend on the sample/can geometry, their scattering powers and experimental setup. Some freely available software, like Ida [18] or Dave [19] is able to do that.

\section{Sample container}

The usual sample container for QENS applications is made of pure aluminium. It has a low scattering and absorption cross section, it is cheap and easy to manufacture. The shape of the container is adapted to the beam size and optimal sample amount (see above): cylinders, double wall cylinders (the sample is between the two walls) or slabs (rectangular). If necessary the sample container can be sealed using indium or lead wires. Cadmium is often used to cover parts of the can to reduce background ( $\mathrm{Cd}$ is toxic, and evaporates already much below its melting point; therefore it should be used with care). For high temperatures other can (quartz, platinum ...), sealing (lead or copper) and shielding materials must be used, if necessary. Most instruments are equipped with standard aluminium cans.

\section{Target systems}

In the following we present some condensed matter systems which have been and are being investigated by means of QENS. The aim is not to give a comprehensive review but rather to give a flavour what problems can experimentally be addressed by means of QENS. We start with the comparatively "simple" metal hydrogen systems, where QENS - especially when single crystals are available enables to elucidate the hydrogen diffusion mechanism in great detail, even for hydrogen in intermetallics. Many fundamental investigations have already been performed and many problems have been solved, so nowadays less people are working in this field. For a future hydrogen economy hydrogen storage systems are required, and light weight metal hydrides like $\mathrm{LiBH}_{4}$ could be a solution to this problem, see the contribution of Remhof et al. in this special volume. To a substantially smaller extent hydrogen has also been studied in proton conducting oxides, which are in some sense more complex systems than metals, and single crystals have not been available for QENS; correspondingly the statements about the diffusion mechanism are less detailed. In melts of metal alloys which form metallic glasses, QENS has been used to look for "glass precursors" in the vicinity of the glass transition; Ni with its large incoherent neutron scattering cross section has been in the focus of interest, a still ongoing research, see the contribution of Demmel et al. in this volume. Other inorganic systems are solid state 
ionic conductors with mobile $\mathrm{Na}, \mathrm{Li}, \mathrm{Ag}$ or $\mathrm{Cl}$ ions; the experimental difficulty is the comparatively weak target ion scattering and the strong host lattice background, and presently less QENS experiments are being performed on theses systems.

Organic, macromolecular and biological systems contain many different hydrogen atoms, and this makes the systems in many cases so complex such that the interpretation of the neutron scattering spectra needs support from molecular dynamics simulations. For organic liquids and for polymers, partial deuteration is a well-established means to make the systems experimentally feasible. Biological systems are often proteins, and they are so complex that QENS spectra cannot be interpreted; only very global statements about the protein dynamics are possible. Often only fixed window scans are performed to determine the mean square displacement of hydrogen atoms. At low temperatures the spatial extent is restricted which is correlated with biological inactivity.

A special case of localized motion is the translational diffusion within a restricted volume; the diffusion, and many other physical and chemical properties in porous media differ from those in the bulk. Porous materials are therefore attractive as novel functional materials.

Concrete and cement is produced all over the world in huge amounts, therefore they have a large economical impact. The performance of these multicomponent systems depends strongly on the mobility of confined water. The paper by Bordallo et al. sheds light on the various aspects of water diffusion in these materials.

Nuclear waste is a very sensitive topic nowadays, and is one of the most important arguments against energy production in reactors. Clays are planned to be part of the barrier system around radioactive waste repositories. The task of such a barrier system is to prevent the escape of radioactive materials into the surrounding for a certain time, until the radioactivity is reduced below a limit. The main transport path is provided by the confined water; therefore it is essential to understand water diffusion in clays. The paper by Malikova et al. in this issue gives an evidence for a 2-dimensional diffusion of water inside the clay interlayer. The method presented in this paper can be used in many porous system to describe the dimensionality of the diffusion.

Finally, confinement is also very important for fundamental research. For example, it is still not yet clear whether there exists a second critical point for water (a liquid -liquid phase transition slightly above $200 \mathrm{~K}$ ). The corresponding point in the phase diagram of water, however, cannot be accessed experimentally due to the fact that around the predicted temperature bulk water is frozen. Inside confinements, however, water (and other liquids as well) can be supercooled, and so it is possible to investigate this "no man's land" in the phase diagram. The article by S.-H. Chen et al. provides arguments for the existence of such a critical point; the academic debate is still open. 


\subsection{Hydrogen in metals}

Many metals dissociatively dissolve hydrogen. The hydrogen atoms occupy interstitial sites and, at low concentration, form a lattice gas. From a practical point of view, this is a very attractive sample system: due to the large scattering cross section of hydrogen QENS spectra exhibit high statistical accuracy. The neutron scattering on $\mathrm{H}$ is mostly incoherent and thus its single-particle diffusional behavior is revealed. From a theoretical point of view this is very interesting due to the light weight of the proton: the classical over-barrier-jumps at high temperatures transform into incoherent tunnelling a lower temperatures and eventually into coherent tunnelling at very low temperatures. Deuterium scatters both coherently and incoherently; consequently in deuterides both the collective and the single-particle diffusional behavior can be studied if one succeeds to separate coherent QENS from incoherent QENS.

The prototype metal hydrogen system is hydrogen in palladium. The host is face centered cubic, and the hydrogen atoms occupy octahedral interstitial sites which form a Bravais lattice. The hydrogen jump diffusion has been studied on a single crystal [20]. The QENS spectra consist of single Lorentzians; the Q dependence of their linewidths in different crystallographic directions could exactly be described in terms of the Chudley-Elliott model. More complex is the situation in niobium which crystallizes in a body centered cubic structure; the hydrogen atoms occupy the tetrahedral interstitial sites, which are not a single interstitial Bravais lattice but a superposition of 6 Bravais lattices. For the interpretation of the QENS spectra on $\mathrm{NbH}_{x}$ the Chudley-Elliott model has been extended to a system of 6 crystallographically different but energetically identical interstitial sites. This would yield 6 Lorentzians in the QENS spectra with certain widths and weights. But the experimental spectra were different and, eventually, could be described in terms of a trapping model (two-state-model): sitting on an interstitial site the hydrogen atom distorts / expands the metal lattice in its vicinity and in this way lowers its potential energy (self-trapping, trapped state); from time to time it escapes, performs a sequence of jumps over the unrelaxed interstitial lattice (free state) until it self-traps again [21]. Hydrogen in niobium doped with interstitial impurities like nitrogen or oxygen shows similar trapping effects [22]; in these (disordered) systems the interstitial sites close to the impurities are energetically lower than those in the undistorted lattice. Regular interstitial lattices with crystallographically and energetically different sites are typical for intermetallics. A well-known example is $\mathrm{LaNi}_{5} \mathrm{H}_{x}$ with $0<x<6$. In order to elucidate the hydrogen diffusion mechanism in this complex system the Chudley-Elliott model has been extended to a system of 9 interstitial sites from which the $6 \mathrm{~m}$ sites form a hexagon in the lattice (perpendicular to $c$, at $c=1 / 2$ ) and the $3 f$ sites in the basal plane with another energy [23]. The QENS experiments have been performed on a single crystal in two different orientations. Only a low hydrogen concentration was allowed, otherwise the brittle intermetallic crystal would have broken. The distorting Ni scattering was removed by substituting 
natural $\mathrm{Ni}$ by ${ }^{60} \mathrm{Ni}$, i.e. by preparing a large $\mathrm{La}^{60} \mathrm{Ni}_{6} \mathrm{H}_{0.06}$ crystal. The hydrogen diffusion consists of a fast localized motion on the hexagon of the $6 \mathrm{~m}$ sites. From time to time the hydrogen manages to jump to a $3 \mathrm{f}$ site in the basal plane and then further to the next hexagon. In a systematic series of experiments Skripov et al. [24,25] investigated Laves phase intermetallic hydrides and could show that depending on the lattice constant (atomic radii of the constituent metals either dumbbell-like or hexagon-like localized hydrogen motions occur in the diffusional process.

\subsection{Fast ionic conductors}

Bare $\mathrm{H}^{+}$ions (protons) are not found in solids under equilibrium conditions $(\tau>$ $\left.10^{11} \mathrm{~s}\right)$. In non-metallic solids $\mathrm{H}^{+}$is always covalently bonded to an electronegative atom or ion in the structure, e.g. O-H or N-H. Usually in solids or liquids the proton is shared between two electronegative ions, e.g. O-H... O, forming a so-called hydrogen bond. Oscillations of $\mathrm{H}$ from one side to the other in a hydrogen bond represent an essential and necessary step in the proton conduction / diffusion process. The second step is the reorientation of the carrier group, which is an oxide anion in proton conducting oxides or a $\mathrm{SO}_{4}$ or $\mathrm{SeO}_{4}$ unit, e.g., in the corresponding salts. In a number of cases there exists a low temperature highly ordered phase with low proton conductivity and at a certain temperature a phase transition to a high-temperature high-conductivity ("superprotonic") phase in which several energetically equivalent sites are possible for the protons. Such a mechanism could, for instance, experimentally be confirmed for $\mathrm{CsHSO}_{4}$ by Beluskin et al. [26] on the basis of high resolution QENS measurements, and in the present volume of Zeitschrift für Physikalische Chemie we refer to the contribution by Krzystyniak on the superprotonic conductor $\left(\mathrm{H}_{3} \mathrm{O}\right) \mathrm{SbTeO}_{6}$. Perovkites like $\mathrm{SrCeO}_{3}$ have to be aliovalently doped, for instance with $\mathrm{Yb}$, in order to become a proton conductor; $\mathrm{Yb}^{3+}$ partially substitutes $\mathrm{Ce}^{4+}$, and for electroneutrality reasons vacancies form in the oxygen sublattice. This system absorbs water from the gas phase with the formation of hydroxyl groups in the oxygen sublattice. QENS measurements on this system [27] showed that the proton diffusion mechanism consists of proton jumps to a neighboring oxygen anion and subsequent reorientation of the hydroxide anions. In addition there are proton trapping effects because the $\mathrm{Yb}$ dopant acts as trap for the proton; the QENS data have been evaluated in terms of a two-state-model, and the relevant jump/ escape rates and jump lengths could be determined.

\subsection{Molten metals}

The transition from a liquid to a glassy state observed in a number of systems and the understanding of the microscopic dynamics involved in these transitions is one of the most challenging problems in condensed matter physics. This glass transition is also observed in some liquid alloys and one of the questions to be answered is the role of the alloy's composition in the glass transition. In many 
binary or ternary melts the cooling rate needed to vitrify the systems is in the order of $10^{6} \mathrm{Ks}^{-1}$, while in alloys like $\mathrm{Pd}_{43} \mathrm{Ni}_{10} \mathrm{Cu}_{27} \mathrm{P}_{20}$ cooling rates less than $0.1 \mathrm{Ks}^{-1}$ are sufficient to bring the alloy into a glassy state [28]. For this special alloy it was found, that the self-diffusivity close to the liquidus temperature as measured by QENS is about one order of magnitude less than for simple metallic liquids at their melting temperature, while on the other hand the diffusivity is larger as compared to good metallic glass formers.

In this issue the article of Demmel et al. provides an introduction into the field of QENS studies in liquid metals. This article also deals with the coherent quasielastic contribution to the measured scattering signal. In [29] the particle dynamics in liquid alkali metals as measured with neutron and X-ray techniques is surveyed and gives a good introduction into the experimental as well as into the theoretical aspects of these metals.

In several experiments metallic liquids have been investigated using containerless techniques, i.e. electromagnetic levitation. In [30] this technique was used to perform QENS experiments on $\mathrm{Ni}_{36} \mathrm{Zr}_{64}$ alloy melts. In this system a large activation energy for Ni self-diffusion was found, resulting from the peculiar short-range order within the Ni-Zr melts. With the same levitation technique $\mathrm{Ni}_{59.5} \mathrm{Nb}_{40.5}$ and $\mathrm{Ni}_{60} \mathrm{Nb}_{34.8} \mathrm{Sn}_{5.4}$ alloys [31], exhibiting an extraordinarily high packing fraction, have been investigated. It was demonstrated that in these alloys the measured self-correlation function shows a slowing down of microscopic dynamics with an increasing packing fraction. These results provide for the first time an experimental value for the critical packing fraction in glass-forming metallic liquids. QENS has also been used to study the self-diffusion of $\mathrm{Ni}$ in liquid $\mathrm{Ni}_{80} \mathrm{P}_{20}, \mathrm{Pd}_{40} \mathrm{Ni}_{40} \mathrm{P}_{20}$ and $\mathrm{Pd}_{43} \mathrm{Ni}_{10} \mathrm{Cu}_{27} \mathrm{P}_{20}$ [32]; these three alloys show very similar packing fraction and the difference in the measured diffusion coefficients is only a slight one. These findings are somewhat unusual with respect to the fact that liquidus temperature and the capability to undercool these systems strongly depend on the composition. On the other hand one can conclude that the packing fraction plays the dominant role and determines the microscopic dynamics and thus the self-diffusion as seen by QENS. The microscopic dynamics in $\mathrm{Cu}_{46} \mathrm{Zr}_{42} \mathrm{Al}_{7} \mathrm{Y}_{5}$ melts was investigated using QENS and it could be shown [28] that in this compound the microscopic dynamics is governed by viscosity which dominates here over packing density.

\subsection{Ionic liquids}

A new class of molten salts, so-called ionic liquids (ILs), with melting points below $373 \mathrm{~K}$, has attracted great interest within the last years. The interest in these materials arises from both science and applications. So far the number of papers dealing with QENS experiments is still limited. Most papers report on experiments on imidazolium-based ILs [33-35] while other cations are far from being investigated. Most ILs are environmentally friendly reaction media, due to the fact that they are nonvolatile, non-inflammable and most of them non-toxic. 
They consist of bulky organic cations and organic or inorganic anions. The imidazolium-based ILs show a variety of phase transition: solid-solid, solid-liquid and smectic $\mathrm{A}$ to liquid. The later transition was found in systems with rather long alkyl chains, namely $\left[\mathrm{C}_{16} \mathrm{mim}\right]\left[\mathrm{PF}_{6}\right]$ and $\left[\mathrm{C}_{14} \mathrm{mim}\right]\left[\mathrm{PF}_{6}\right][55]$. In [34] the system $[\mathrm{bmim}]\left[\mathrm{PF}_{6}\right]$ was investigated using QENS. In this IL two different relaxation processes could be identified: a fast $\beta$-process inside a cage-like region and a slower $\alpha$-process. The fast process shows neither a Q- nor a temperaturedependence whereas the slow $\alpha$-process reveals a non-Arrhenius temperaturedependence and approximately a Gaussian Q-dependence. Using inelastic fixed energy scans it could be shown that at low temperatures the butyl and methyl groups relax according to a Debye-like process while at temperatures above the glass-temperature these alkyl chains show a diffusional behavior represented by a stretched exponential (due to a distribution of relaxation times). Due to the fact that in all mentioned papers the authors analyzed the QENS-data in time-space rather than in energy domain no information about the geometry of the relaxational dynamics was presented.

In a recent publication [36] the protic ionic liquid N,N,N',N'-tetramethylguanidium bis(perfluoroethylsulfonyl)imide, a system with the possible application as electrolyte in proton-conducting fuel cells, was investigated. In this system four different dynamic processes have been found: rotation of the methyl groups and a localized process that was attributed to the protons in the $\mathrm{NH}_{2}$-groups. These two processes take place both in the solid and in the liquid phase. Above the melting temperature two further processes have been detected both with translational character. The slower one of these two relaxations was identified as an unrestricted translational diffusion while the faster one shows up signatures of confined translation diffusion.

\subsection{Polymers}

In the last decades the research on polymers was mainly focused on the understanding of the motion of entangled polymers. For non-entangled linear polymers the viscoelastic stress relaxation can be described within the Rouse-model (for a detailed survey of polymer dynamics and its statistical descriptions see [37]). The situation for entangled branched polymers is more difficult to describe. Even more challenging is the situation for entangled ring polymers; new observations and models are discussed in [38]. The combination of MD simulation techniques and scattering experiments plays also in polymer research an increasing role. In [39] both techniques have been applied to a poly(ethylene oxide) homopolymer system above the melting temperature. It is demonstrated that the force field used for the MD simulations is exact in the sense that it reproduces the measured data. With this knowledge further physical parameters, which are not accessible in experiments, have been extracted from the MD trajectories. In [40] the authors derived models of the local $(\sim 0.1 \mathrm{~nm})$, short-time $(\sim 10 \mathrm{ps})$ dynamics of linear polymers. This model takes into account orientational diffusion along the poly- 
mer chain, local conformational transitions and long-time, large-scale motions. The same model is applied to QENS data obtained on highly entangled polyethylene oxide (PEO) melt at $373 \mathrm{~K}$.

The self-diffusion in medium-chain n-alkanes (ranging from $\mathrm{C}_{8} \mathrm{H}_{18}$ to $\mathrm{C}_{54} \mathrm{H}_{110}$ ) was investigated using QENS and pulsed field gradient nuclear magnetic resonance (PFG-NMR) [41]. These alkanes are small enough to be far away from the Rouse regime. The experimental data as obtained by using TOF-QENS have been analyzed by a model function consisting of two Lorentzians. These two Lorentzians describe both the long-range diffusion of the whole molecule and some local molecular motions of the alkanes. A comparison of the diffusion coefficients deduced with QENS on the one hand (short-time diffusion coefficients) and with PFG-NMR on the other hand (long-time diffusion coefficients) showed that the values are clearly drifting apart. The reason for this fact is attributed to the crossover of some/several diffusive processes with very similar relaxation times (diffusion QENS-models with different diffusion coefficients). In [42] the dynamical processes in liquid $\mathrm{C}_{32} \mathrm{H}_{66}$ have been further investigated by performing resolution resolved QENS experiments. For all observation times the QENS-signal is dominated by a processes having a $\mathrm{Q}^{2}$-dependent linewidth and an apparent diffusion coefficient $D_{a}\left(t_{0}\right)$ which depends on the observation time $t_{0}$. The apparent diffusion coefficient $D_{a}\left(t_{0}\right)$ is interpreted as a description of considerably different molecular dynamical processes which could be identified by analyzing data from corresponding MD simulations. Methyl group dynamics plays an important role in many polymer systems. In [43] this dynamical process is explained in great detail and provides a good starting point for the phenomenon of the so-called tunneling. In addition, the number of reports on experiments on micellar systems is increasing continuously. In this issue the papers of Holderer et al. and Sharma et al. deal with those solutions and their dynamical properties as seen by QENS. A second paper by Sharma et al. deals with the dynamical processes of short hydrocarbons in zeolites.

\subsection{Bio systems}

Systems forming the broad field of biologically relevant species, i.e. proteins, cells and so on are even much more complex with respect to their dynamical behavior and processes occurring. Thus it is a quite challenging task to study these kinds of systems. Very often but not solely, the research on these systems is restricted to the investigation of the mean square displacement. A review on the current research on dynamical processes on biological systems can be found in the article of A. Stadler in this issue. Furthermore the article of Natali et al. in the current issue deals with protein dynamics and the formation of amyloid fibrils and its connection to diseases like Alzheimer. Water plays an essential role in biological systems; therefore the dynamics of water, especially its dynamical properties in confinements and its influence on the functionality of biological systems is in the focus of current research activities. The paper of Chen et al., 
also in the current issue, deals with the properties of water and supercooled water in 3-D and 1-D confinements.

Gaspar et al. [44] investigated the interplay between structure and dynamics of different proteins using TOF-techniques. They scrutinized casein proteins (belonging to the class of natively disordered proteins) and in addition three wellfolded proteins with distinct secondary structures (myoglobin, lysozyme and concanavalin A). The results obtained show differences in the motional amplitudes of well-folded proteins, i.e. the $\beta$-sheet structures appear to be more rigid than a cluster of $\alpha$-helices.

In [45] the influence of hydration water on the dynamics of side chains of hydrophobic peptides was probed by elastic and quasielastic neutron scattering experiments. When going from the dry peptide to the highly hydrated situation (but before the liquid state is reached) the authors found that the confined translational diffusion of long chains turns up, but only if the level of hydration is high enough to form a 3-dimensional network of water molecules. On the contrary for lower hydration levels only rotational motions (of the methyl groups) have been observed. On the basis of the results presented in this paper the authors reason that the onset of a dynamical transition and large amplitude motions should be considered as a physical characteristic of biomolecules related to hydration water and independent of the function of the protein involved.

Another field of research is related to the behavior of water in living cells and the role of water with respect to the functionalities of these cells. It is now well known that close to the body temperature of different species there is a transition in hemoglobine $(\mathrm{Hb})$ behavior. This was demonstrated with macroscopic method like for example micropipette aspiration experiments. In [46] quasielastic neutron scattering has been used to investigate the molecular origin of the transition in $\mathrm{Hb}$ in whole human red blood cells (RBCs) in vivo. The experiments have been performed as a function of temperature and show that a change in the geometry of the internal protein motions take place at $36.9{ }^{\circ} \mathrm{C}$, thus at human body temperature. Furthermore, indications of a partial unfolding of the protein at this temperature could be found. To summarize, these results clearly demonstrate the connection between changes on a molecular level (in space and time) and microscopic effects in whole RBCs.

From the above delineated effect and the correlation between the transition temperature and body temperature for $\mathrm{Hb}$ from different species, one can reason that this effect might be important for the biological function. To sheet more light on the relevance of internal protein dynamics for the biological functionality, the internal motions have been studied with elastic ('fixed-window scans') as well with quasielastic neutron scattering. The experiments have been done for systems ranging from fully hydrated protein powder to concentrated $\mathrm{Hb}$ solutions. The experimentally observed data clearly show that a certain degree of hydration is needed to show up with a transition in protein dynamics. Finally we mention the paper by Biehl et al. [47]. This paper reports on the direct experimentally observation of the domain dynamics of the protein alcohol dehydrogenase ADH. 


\section{References}

1. T. Springer, Quasielastic Neutron Scattering for the Investigation of Diffusive Motions in Solids and Liquids. Springer Tracts in Modern Physics. Springer, Berlin (1972).

2. M. Bée. Quasielastic Neutron Scattering: Principles and Applications in Solid State Chemistry, Biology and Materials Science. Adam Hilger, Bristol (1988).

3. R. Hempelmann. Quasielastic Neutron Scattering and Solid State Diffusion. Oxford Science Publications (2000).

4. R. E. Lechner and S. Longeville. Neutron Scattering in Biology. Springer (2005) chapter 15, pages 309-354.

5. G. Schatz and A. Weidinger. Nukleare Festkörperphysik. Teubner (1992).

6. W. G. Williams. Polarized Neutrons. Clarendon Press, Oxford (1988).

7. S. Koizumi and T. Inami, Macromolecules 32 (1999) 5613.

8. C. T. Chudley and R. J. Elliott, Proc. Phys. Soc. London 77 (1961) 353.

9. P. L. Hall and D. K. Ross, Mol. Phys. 42 (1981) 673.

10. K. S. Singwi and A. Sjölander, Phys. Rev. 119 (1960) 863.

11. L. Liang, R. Rinaldi, and H. Schober. Neutron Applications in Earth, Energy and Environmental Sciences. Springer (2009).

12. P. Böni, Physica B 279-278 (2000) 6.

13. R. Zorn, Nucl. Instr. Meth. Phys. Res. A 603 (2009) 439.

14. http://www.ill.eu/other_sites/BS review/index.htm.

15. F. Mezei, C. Pappas, and T. Gutberlet, editors. Neutron Spin Echo Spectroscopy / Basics, Trends and Applications. Lecture Notes In Physics. Springer, Berlin (2003).

16. http://www.ill.eu/top links/publications/.

17. http://idb.neutron eu.net/instrument.php.

18. http://iffwww.iff.kfa-juelich.de/ wuttke/doku/doku.php?id = frida:frida1:fr

19. www.ncnr.nist.gov/dave/.

20. J. J. Rush and J. M. Rowe, Phys. Rev. B 9 (1974) 5039.

21. V. Lottner, H. R. Schober, and W. J. Fitzgerald, Phys. Rev. Lett. 42 (1979) 1162.

22. D. Richter and T. Springer, Phys. Rev. B 18 (1978) 126.

23. C. Schönfeld, R. Hempelmann, D. Richter, T. Springer, A. J. Dianoux, J. J. Rush, T. J. Udovic, and S. M. Bennington, Phys. Rev. B 50 (1994) 853.

24. A. V. Skripov, J. C. Cook, T. J. Udovic, V. N. Kozhanov, and R. Hempelmann, Appl. Phys. A 74 (2002) 948.

25. A.V. Skripov, M. A. Gonzalez, and R. Hempelmann, J. Phys.: Condensed Matter 20 (2008) 085213.

26. A. V. Beluskin, C. J. Carlile, and L. A. Shuvalov, J. Phys. C: Condensed Matter 4 (1992) 389.

27. Ch. Karmonik, Th. Matzke, R. Hempelmann, and T. Springer. 50a (1995) 539.

28. S. M. Chathoth and A. Podlesnyak, J. Appl. Phys. 103 (2008) 013509.

29. W.-C. Pilgrim and Chr. Morkel, J. Phys.: Condens. Matter 18 (2006) R585.

30. D. Holland-Moritz, S. Stüber, H. Hartmann, T. Unruh, T. Hansen, and A. Meyer, Phys. Rev. B 79(6) (2009) 064204.

31. S. M. Chathoth, B. Damaschke, M. M. Koza, and K. Samwer, Phys. Rev. Lett. 101(3) (2008) 037801.

32. S. M. Chathoth, A. Meyer, M. M. Koza, and F. Juranyi, Appl. Phys. Lett. 85(21) (2004) 4881.

33. J. De Roche, C. M. Gordon, C. T. Imrie, M. D. Ingram, A. R. Kennedy, F. Lo Celso, and A. Triolo, Chem. Mater. 15(16) (2003) 3089-3097.

34. A. Triolo, O. Russina, V. Arrighi, and F. Juranyi, J. Chem. Phys. 119(16) (2003) 8549-8557.

35. A. Triolo, O. Russina, Ch. Hardacre, M. Nieuwenhuyzen, M. A. Gonzalez, and H. Grimm, J. Phys. Chem. B 109 (2005) 22061. 
36. E. Mamontov, H. Luo, and S. Dai, J. Phys. Chem. B 113 (2009) 159.

37. M. 37 and S. F. Edwards. The Theory of Polymer Dynamics. Oxford University Press (1988).

38. M. Kapnistos, M. Lang, D. Vlassopoulos, W. Pyckhout-Hintzen, D. Richter, D. Cho, T. Chang, and M. Rubinstein, Nature Materials 7 (2008) 997.

39. M. Brodeck, F. Alvarez, A. Arbe, F. Juranyi, T. Unruh, O. Holderer, J. Colmenero, and D. Richter, J. Chem. Phys 130 (2009) 094908.

40. J.-M. Zanotti, L. J. Smith, D. L. Price, and M.-L. Saboungi, J. Phys.: Condens. Matter 18 (2006) S2391-S2402.

41. Ch. Smuda, S. Busch, G. Gammecker, and T. Unruh, J. Chem. Phys. 129 (2008) 014513.

42. T. Unruh, Ch. Smuda, S. Busch, J. Neuhaus, and W. Petry, J. Chem. Phys. 129 (2008) 121106.

43. J. Colmenero, A. J. Moreno, and A. Alegria, Prog. Polym. Sci. 30 (2005) 1147.

44. A. M. Gaspar, M.-S. Appavou, S. Busch, T. Unruh, and W. Doster, Eur. Biophys. J. 37 (2008) 573.

45. D. Russo, J. Teixeira, and J. Ollivier, J. Chem. Phys. 130 (2009) 235101.

46. A. M. Stadler, I. Digel, G. M. Artmann, J. P. Embs, G. Zaccai, and G. Büldt, Biophys. J. 95 (2008) 5449.

47. R. Biehl, B. Hoffmann, M. Monkenbusch, P. Falus, S. Préost, R. Merkel, and D. Richter, Phys. Rev. Lett. 101 (2008) 138102.

48. $1 \mathrm{meV}=1.60219 \cdot 10^{-22} \mathrm{~J}$

$=11.6045 \mathrm{~K}$

$=8.0668 \mathrm{~cm}^{-1}$

$=0.09648 \mathrm{~kJ} \mathrm{~mol}^{-1}$

49. Data are collected as a function of time with the periodicity of the chopper system. Frame overlap occurs if the periodicity time is too small, and so the end of one frame and the beginning of the next frame is collected in the same time channels.

50. Mosaicity is the angle which measures the goodness of the orientation of the crystallites. If this is too small (i.e. the crystal is too perfect), intensity is lost, if too big, the energy resolution will be worth.

51. $\gamma$-rays are a form of electromagnetic radiation of short wavelength (energies above $100 \mathrm{keV}$ ). Because they are a form of ionizing radiation, gamma rays can cause serious damage when absorbed by living tissue, and are therefore a health hazard.

52. Angular measure of the increase of beam size with distance due to different flight directions of the neutrons, which are not exactly parallel.

53. Borplastic is a polymeric, rubber like material to which natural Boron has been added in order to absorb neutrons.

54. The energy of the incoming and scattered neutrons are fixed and so the amount of elastically (or sometime inelastically, within a certain energy window) scattered neutrons are measured usually only as a function of temperature.

55. The notation $\mathrm{C}_{n}$ represents the alkyl chain $\mathrm{C}_{n} \mathrm{H}_{2 n+1}$, whereas mim and bmim are short forms for methyl- and butyl-methyl-imidazolium 